\title{
A Computation and Control Architecture of Virtual Manufacturing Shop
}

\section{Zhiming Wu}

Dept. of Automation, Shanghai Jiaotong University

E-mail: ziminwu@mail.sjtu.edu.cn

Keywords Virtual Manufacturing, FMS, Petri net modelling, Monitoring \& Control

\begin{abstract}
Virtual manufacturing (VM) is a model for an integrated application of manufacturing system. In this paper, only the information infrastructure of manufacturing shop is considered. The system architecture, modelling and analysis, include simulation and control software, can be tested and operated on a platform of distributed computer system (DCS). Generally, a client/server LAN with enough attached measurement/control terminals would be sufficient to imitate all the mechanisms and activities of such a manufacturing information system. A more extensive concept of VM that may include virtual product designing and virtual business operating is not covered in this work.
\end{abstract}

\section{INTRODUCTION}

Modern manufacturing system, such as flexible manufacturing system (FMS), has spent a large amount of enterprise budgets during its construction. Therefore, as soon as it is built, achieving a complete and efficient use of the whole system resources is especially important to quickly create more benefits and then compensate the huge investments.

Among all the technical resources of a manufacturing system, the information sub-system corresponds to the processes of message communication, functional computation and behavior control. They would not be the most expensive part in FMS, but they do give great influences to the normal and effective use of the whole facilities.

Since equipment and component of FMS are supposed to be stable and reliable, for instance the NC machines and tools are well developed nowadays, therefore, constructing high quality functional modules in the information system is of particular importance to make all facilities to be 
used in a coordinating/cooperating environment. Besides, approaches to check the correctness of all the operations in global FMS should be very well established.

\section{ARCHITECTURE OF DISTRIBUTED FMS INFORMATION SYSTEM (IS)}

Like many Communication-Computation-Control (C-C-C for short) Integrated Systems used in the application areas, FMS performs its activity within a fully distributed environment. Many of active resources in a FMS may play their roles independently in the real time. Different entities in a FMS can be classified by concepts of objects. For some active and autonomous ones among them, the name of actors or agents is always used.

Considering of information system (IS) in FMS simulation, although all the main C-C-C activities are able to completed by a powerful processor via a multi-thread operation system, a distributed computer system may describe the module structure and the inter-processes relations more accurately. In particular, when the message or event occurring concurrently in the real time, a fixed access policy of computer operation system thread might not be able to give the C-C-C time responses correctly because of competition of between them. Besides, as the simulation of each subsystem contains some more detail task to do, an independent processor may provide more neat software and I/O interface to handle quite a lot of requirements within a short time duration. Thus, a distributed computer system (DCS) should be introduced to represent all the system behaviours within such an information scheme.

Suppose the shop-controller, the material handling system (MHS) and each workstation (WS) are represented by a set of independent processors. Then, the DCS is given in Fig. 1.

From the idea of object-oriented programming (OOP) [Cor 1990, Boo 1994], it is able to form several real-entity classes to represent different kinds of real entities in the FMS. Among them, some active objects attached with their private methods, algorithms and data/knowledge may complete decision and control works separately in real time.

Work-pieces (WPs) in FMS haven't been clearly shown in the diagram. However, from the view of communication network, they actually play the roles of message-package flow transferred through the lower right side in the figure 1. MHS becomes a "loaded channel" or a "dynamical router" that transports WPs guided by the attached message code of package within the FMS "network". 


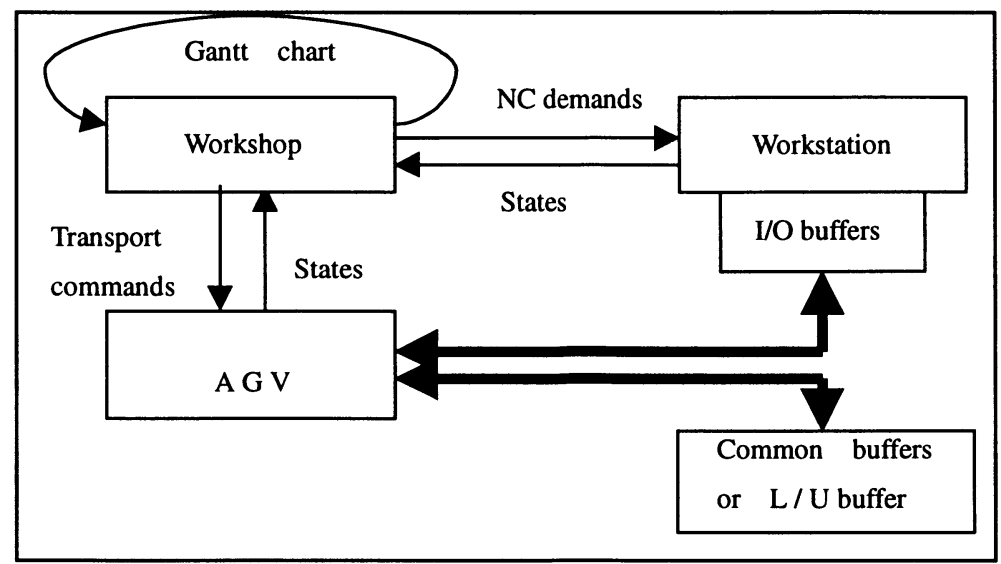

Fig. 1, Information processing within different resources in FMS

The codes attached with each WP may be considered as a "protocol" information that can be understood by MHS. They indicate the WSs where the WP can be picked up/put down and how it can be processed. Thus, during the IS modelling, a WP do need to keep its private information (like an object encapsulates its main features in OOP) such as name, state and special processing routes to show its existence, states and the expected next treatment.

All the WSs keep a rather simple and similar working cycle. Inspiring of their different types, each WS has its own private information codes that can be identified by other objects via its exchange interface. The terminal named "input-stand" on WS is used for accepting WP, and "output-stand" as a peer for sending WP. The machining loop of a WS consists of the following steps:

(1) After checking, take a right WP from the input-stand of WS.

(2) Clamp (fix) WP at the right position and changing cutter in the same time.

(3) Call the NC programming, start machining and finally push the processed WP to the output-stand of WS. Wait to be picked away.

The MHS completes the task of passing WPs (also the attached codes) within different machining loops (similar to message interleaving within DCS). Its main function includes:

(1) Take commands from shop-controller, choose the right WP, set up the picking-up position and releasing position for this WP.

(2) Ride MHS (AGV) along the track, stop AGV at predefined points, load/unload the WP towards different kinds of buffer area.

(3) Keep the WIP (work in process) in a queue for next processing. 
The general shop-controller plays several very important roles in the top level, including:

(1) Receive the new batch data, provide several manufacturing schedules in off-line.

(2) Simulate these scheduling programs within the available shop model (discussed in next section), choose one of them based on their performance evaluations.

(3) Control the WP flow at the I/O terminal of FMS, monitor buffer capacity and machining quality to avoid deadlock, collision and other conflicts occurring on-line.

(4) Take the system state-messages within FMS and also its environment, especially the failure signal of tool or machine, decide if a rescheduling is needed in this moment.

\section{SPECIFICATION AND MODELLING OF FMS VIA PETRI NET}

Mainly, there are 4 classes of entities existed in a manufacturing system: WS, WP, buffer, and MHS. Several kinds of MHS can be used in the FMS, such as robot, mechanical arm and/or automated guided vehicles (AGV). Here, only an AGV is considered. Besides, there are many styles of buffer in an FMS, for instance, a single common buffer aside the AGV track, the input/output stand of WS or the load/unload area of the shop. Physically, the WP in FMS is not fully free. It should be attached with other entities in the system, existing as WP in WS, WP in buffer or WP in AGV.

In accordance with the system behavior, 4 classes of objects are defined in the FMS. By using the Unified Modelling Language (UML), the corresponding attributes and methods of these objects, and also the relation between these object classes can be explained in a special diagram (that is neglected because of limited space) [Rat 1999].

Let each operation of a WP is defined as a job. Then, for a general " $n$ WPs-m WSs" FMS production problem, the process planning of a given WP type defines a set of jobs handling by different WS resources in accordance with a specified sequence (route). On the other hand, for a definite WS, there is a set of WPs that can be accepted to form a service chain during the same batch task. From the system analytical view, FMS belongs to the discrete event systems (DES) and keeps the feature of asynchronous concurrency. Therefore, control mechanisms based on Petri net model are preferred by most of the authors. 


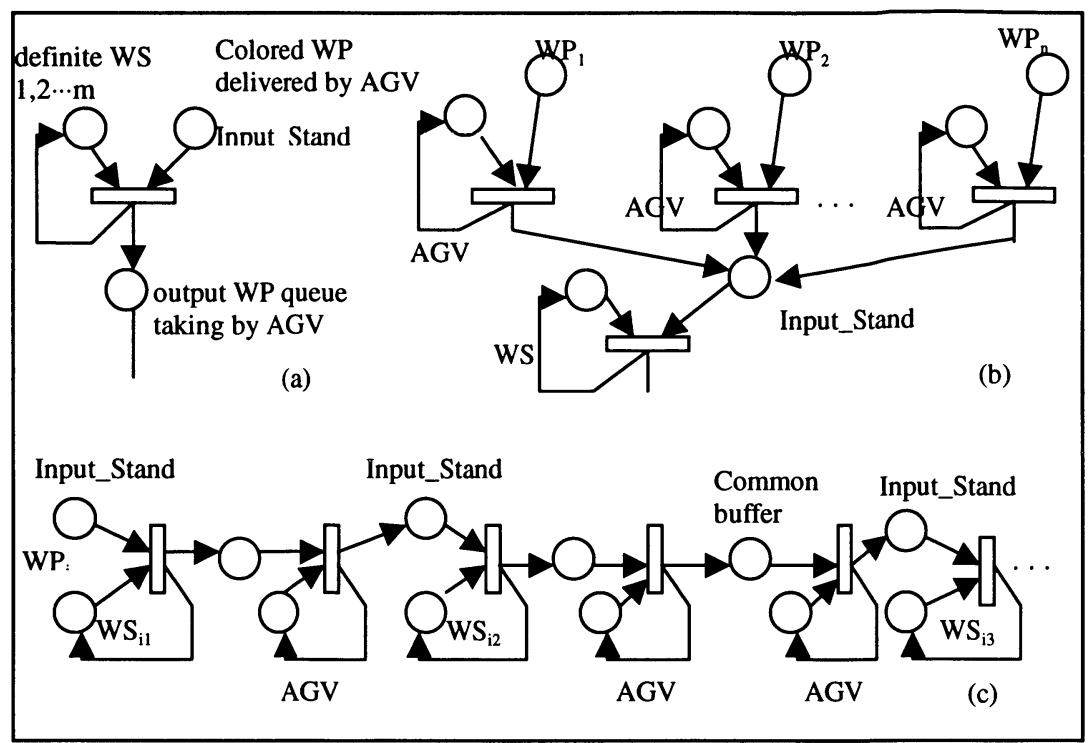

Fig. 2, (a) processing unit, (b) queue waiting for WS service, (c) WP processing sequence

Suppose different codes have been defined for identifying all kinds of WPs and WSs. According to object-oriented Petri net or high level Petri net [Jaf 1992, Wang 1996, Chen 1997], we may identify these differences with attributes of object or their "colored" codes.

A colored WP is going to be processed if it has been put into an inputstand of a "free" WS (ready to work). As soon as a job has been processed, the WP would be put to the output-stand immediately if it is empty. Then, it is waiting to be transferred to other buffer (maybe a common buffer or an input-stand of a WS) by AGV.

As an elementary job-processing unit, the queue of WPs waits for a service to be given by resource WS. The processing sequence of a given type WP during the batch can be shown in figure 2(a), (b), and (c) respectively. At the same time, a Petri net model that denotes AGV connecting all of WS loops and realising inter-loop message passing between them is shown.

Based on these sub-models, a general model of FMS can be summarized, that may combine all the activities and behaviors of the main resources, and provide a complete dynamic view for the coupled processes in whole system.

In accordance with the model framework of a FMS, several successful FMS simulation tools built on the language of OOP (C++, UML) or various Petri Net Tools have been developed nowadays [Wang 1996, Chen 1997, Qu 1999]. The modular structure of software makes the components can be 
reused for different batch tasks and shops. It is really not difficult to follow the heterogeneous routing traces of all the WPs in a FMS [Wu 1999].

However, the modelling efforts listed above can only create an environment to test the FMS freely. To make an efficient use to prove the potential ability of FMS, one must keep a criterion-guided (simply, benefitdirected) control strategy, that is realized by controller's command-sequence to obtain an optimal simulation result. That means different controllers must be used to supervise both the activities of WP transportation and job processing to ensure the whole system working normally and get a fairly good performance finally.

\section{MONITORING AND CONTROL IN THE INTELLIGENT MANUFACTURING SYSTEM}

Some authors have pointed out that the monitoring and control structure of the FMS shop floor is often constructed in a hierarchical way. That means there are many monitors and controllers positioned in different system level supervising respective objective aims. They can be specified from a threshold index, a critical value or an event (Boolean action) in a definite point to the general view considering the states of whole shop.

Among all these tasks, the control blocks in equipment level are relatively mature, such as PLC and continuous variable controllers. However, their monitoring usually require some special techniques/instruments to help high precision measurement and control, such as pattern recognition of a physical entity, shape characteristics extraction sensor, feature classifier and some non-parametric clustering algorithms.

Besides some special hardware instruments, the information structure of the monitoring system may build on a general artificial intelligence (AI) and sensor-fusion platform that combines measuring data, training data, knowledge and pictures of WPs/cutters positioned on the equipment and some respective algorithms. Although it is important for WSs and AGVs in real cases, from the top view of a VM system, their influences are local. They can be designed and tested separately.

The monitoring tasks in the co-ordination and decision level usually need rather simple instruments. However, high level monitoring emphasises on providing enough information for shop controller. Many indices have to be kept for monitoring at every moment/step to assure the system free from blocking. Some important factors must be followed up-to-date, including the number of WIP remaining in the shop, the number of free common buffer, the length of waiting queue for different WSs, the total throughput time of different WPs, etc. Based on such a high level monitoring, a global-shop 
diagnosis can be made by shop controller that must predict and solve all the faults and mistakes in an early and harmless stage.

Control problems at these levels concentrate on how to create a stable and reliable environment for the effective use of FMS. Firstly, it must make use of the Gantt chart and the records of real-time state monitoring to manage the behaviours of the whole system.

Static scheduling is an off-line task completed by high-level shop controller to arrange the WPs entering/processing sequence for different WSs. According to the schedule, Gantt chart is obtained as a reference for whole operation process to give the detail process step by step graphically. However, Gantt chart can't be precisely executed in practical shop in most cases. There is always a time deviation between the execution records and Gantt chart. Therefore, monitoring high-level states sounds important in giving a diagnosis to make sure if a deviation can be absorbed in its propagation or it makes system to crash few steps later, and checking if all the performances of the system are satisfied or accepted. That means a highlevel controller takes care of the performances of whole FMS. In the normal cases, it keeps as small deviation with the Gantt chart to make FMS work in order and in a high efficiency. In an abnormal case, it follows the control strategy to keep the system free from deadlock, at least it may sure that the blocking state can be recovery from an accident very quickly.(even in rescheduling)

Besides making Gantt chart in the off-line case, adjusting input interval between WPs is one of the main activities of shop controller. The problem is when too many WPs allowed entering, the system might be blocked, but less input always causes low production efficiency and also low resources utilities. Since the information structure of FMS belongs to a fairly large and distributed discrete event system, a formal model and powerful numerical algorithm should be considered. The typical problem that need to be solved in high-level is generally with analytical approach or heuristics, that contains:

(1) An algorithm to make static/dynamic scheduling, re-scheduling and AGV transportation scheduling subject to a criterion considering production benefit [Zhao 2001].

(2) An algorithm to manage and control the time length/interval of $1 / O$ queues of material flow subject to both production effectiveness and safeness [Ram 1995, Mur 1986].

The high performance properties expected by modern manufacturing systems encourage people to develop more advance techniques/methods for practical uses. Nowadays, many mathematical programming methods (including operations research OR), soft-computing algorithms (including 
evolutionary, genetic, simulated annealing and fuzzy) and $\mathrm{AJ} /$ expert systems (AI/ES) have been used to establish a complicated algorithm-base for solving these problems [Baker 1998]. The information structure of high-level problem-solvers is shown in figure 3. For a FMS input controller, its algorithm has to work in parallel with the modules of simulation and monitoring to obtain the results for them in on-line uses.

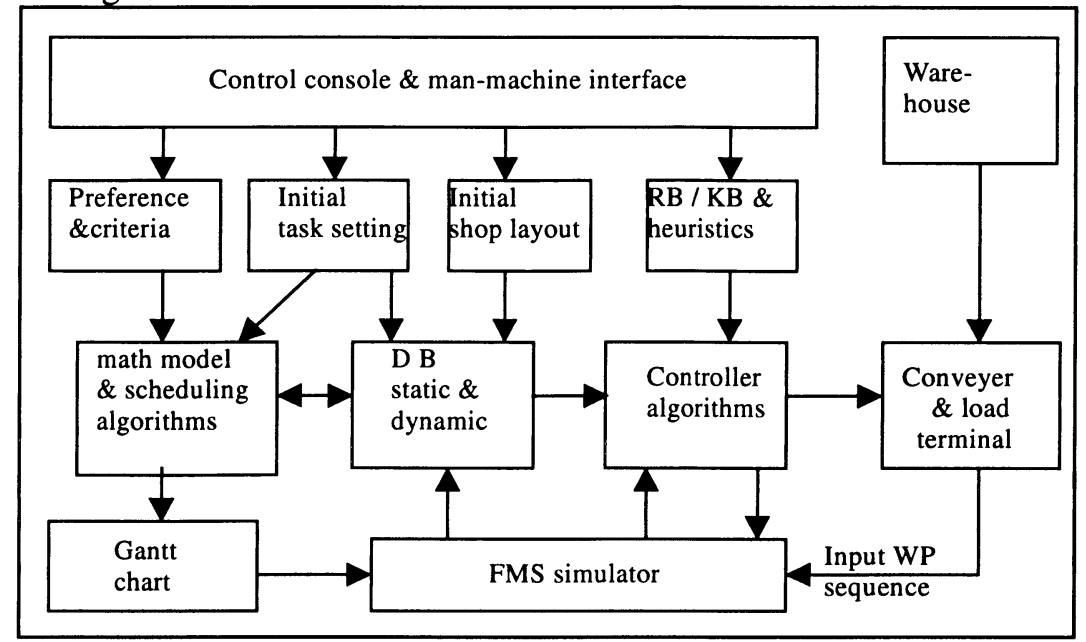

Fig. 3, Information structure of scheduler and controller in a FMS

Although different approaches have been employed for different monitoring and control problems, there are still many resources and information must be shared by those problem-solvers. Some obvious ones are:

(1) Static/dynamic database storing documents of shop resources, batch task and operation records,

(2) Rule-base/knowledge-base and the inference mechanisms that may generate many new conclusions according to the up-to-date system states in dynamic DB,

(3) Data/knowledge and their various reasoning results achieved for solving one special problem in real time would be useful for other problemsolvers' reference.

It means a blackboard architecture [McM 1996] of manufacturing systems is helpful.

\section{CONCLUSION}

The paper gave a complete information structure for a modern manufacturing shop. From the structure model of main components to the overall system, a formal model is given for specifying the main requirements 
of FMS. Several realization approaches and techniques for the virtual FMS, such as its simulation, monitoring and control are discussed.

Corresponding to the practical needs of objective system and the technical features of products in both hardware/software, an experienced designer may decide the number of functional modules which should be integrated together for the given project. It is worth noting that some modules recommended in this paper are still being developed from the practical and commercial view. However, the rapid advances of IT, AI, SE, and also the computer/mechatronic devices and techniques would much improve the abilities in solving most of these problems. An ideal, fully automated and person-less VM shop can be realised quickly.

\section{REFERENCES}

[1] Baker, A.D., A survey of factory control algorithms that can be implemented in a multiagent heterarchy: dispatching, scheduling and pull, Journal of Manufacturing Systems, Vol.17, No.4, 1998, pp297-319

[2] Booch, G., Object-Oriented Analysis and Design with Applications, Benjamin/Cummings, 1994

[3] Chen, K. and S.Lu, A Petri net and entity-relationship diagram based object-oriented design method for manufacturing system control, Inter. Journal of Computer Integrated Manufacturing, Vol. 10, No. 1, 1997, pp 14-28

[4] Coad, P. and E.Yourdon, Object-Oriented Design with Applications, Benjamin/Cummings, 1990

[5] Jafari, M., An architecture for a shop floor controller using colored Petri nets, Inter. Journal of Flexible Manufacturing Systems, Vol. 4, No. 2, 1992, pp 159-182

[6] McManus, J.W.and W.L.Bynum, Design and analysis techniques for concurrent blackboard systems, IEEE Tr. on System, Man and Cybernetics, part A, Vol. 26, No.6, 1996, pp669-680

[7] Murata, T., N.Komoda, K. Matsumoto and K. Haruna, A Petri net based controller for flexible and maintainable sequence control and its application in factory automation, IEEE Trans. Industrial Electronics, Vol. 33, No. 1, 1986, pp 1-8

[8] Qu-yang, C., Developing a Petri net based simulation model for a modified hierarchical shopfloor control framework, Inter. J. of Production Research, Vol. 37, 1999, pp31393167

[9] Raman, Narayan, Input control in job shops, IIE Tr. Vol.27, 1995, pp 201-209

[10] Rational Software about Rational ROSE, http ://www.ration.com/UML/Rosel.html 1999

[11] Venkatesh, K. and M.C.Zhou, Object-oriented design of FMS control software based on object modelling technique diagram and Petri nets. Journal of Manufacturing Systems, Vol. 17, No. 2, 1998, pp 118-136

[12] Wang, L., Object-oriented Petri nets for modelling and analysis of automated manufacturing systems, Computer Integrated Manufacturing Systems, Vol. 26, No. 2, 1996, pp 111-125

[13] Wu, Z., CEM/T nets, a high level Petri net for FMS modelling, Inter. Journal of Intelligent Control and Systems, Vol. 3, No. 3, 1999, pp 377-387

[14] Zhao, C. and Z.Wu, A genetic algorithm approach to flexible scheduling problems in FMS, will appear in Inter. J. of Flexible Manufacturing Systems, Vol. 13, No. 1, 2001 